\title{
Selection on stability across ecological scales
}

\author{
Jonathan J. Borrelli ${ }^{1}$, Stefano Allesina ${ }^{2}$, Priyanga Amarasekare ${ }^{3}$, Roger Arditi ${ }^{4}$, \\ Ivan Chase ${ }^{1}$, John Damuth ${ }^{5}$, Robert D. Holt ${ }^{6}$, Dmitrii O. Logofet ${ }^{7}$, Mark Novak ${ }^{8}$, \\ Rudolf P. Rohr ${ }^{4}$, Axel G. Rossberg ${ }^{9}$, Matthew Spencer ${ }^{10}$, J. Khai Tran ${ }^{11}$, and \\ Lev R. Ginzburg ${ }^{1}$
}

${ }^{1}$ Stony Brook University, Stony Brook, NY, USA

${ }^{2}$ University of Chicago, Chicago, IL, USA

${ }^{3}$ University of California Los Angeles, Los Angeles, CA, USA

${ }^{4}$ Department of Biology, University of Fribourg, Fribourg, Switzerland

${ }^{5}$ University of California Santa Barbara, Santa Barbara, CA, USA

${ }^{6}$ University of Florida, Gainesville, FL, USA

${ }^{7}$ Laboratory of Mathematical Ecology, A.M. Obukhov Institute of Atmospheric Physics, Academy of Sciences, Moscow, Russia

${ }^{8}$ Oregon State University, Corvallis, OR, USA

${ }^{9}$ Centre for Environment, Fisheries, and Aquaculture Science (CEFAS), Lowestoft, UK

${ }^{10}$ University of Liverpool, Liverpool, UK

${ }^{11}$ Syngenta, Greensboro, NC, USA

\begin{abstract}
Much of the focus in evolutionary biology has been on the adaptive differentiation among organisms. It is equally important to understand the processes that result in similarities of structure among systems. Here, we discuss examples of similarities occurring at different ecological scales, from predator-prey relations (attack rates and handling times) through communities (foodweb structures) to ecosystem properties. Selection among systemic configurations or patterns that differ in their intrinsic stability should lead generally to increased representation of relatively stable structures. Such nonadaptive, but selective processes that shape ecological communities offer an enticing mechanism for generating widely observed similarities, and have sparked new interest in stability properties. This nonadaptive systemic selection operates not in opposition to, but in parallel with, adaptive evolution.
\end{abstract}

\section{An explanation for ecological similarities}

In recent years, there has been a great deal of ferment focused on the integration of ecology and evolution [1]. Evolutionary and ecological processes can generate a variety of community configurations, but some are observed in nature more or less frequently than others. Not all of these configurations will be equally feasible (see Glossary) or dynamically stable, such that all member species can persist in sufficiently high abundances that extinction of any one is unlikely. When interpreting broad patterns in ecology, we must account for feasibility and stability. Feasible and stable configurations should be observed in

Corresponding author: Borrelli, J.J. (borrejj@gmail.com).

Keywords: stability; feasibility; selection; macroecology. nature with a higher frequency than configurations that are strongly intrinsically unstable.

If there is no way for a given set of species to coexist (not feasible), then the configuration is doomed to extinction. For example, in the well-studied intraguild predation module, an intraguild predator and its prey (both of whom share a third resource) are unlikely to coexist if both predators are equal competitors for the shared resource and not limited by other factors, such as interference competition (Box 1). Instead, we should only expect to observe this module when the intraguild prey is able to outcompete its predator for the shared resource [2-4]. In other cases, a feasible equilibrium does exist but is intrinsically unstable.

This example illustrates what we suggest to be a more general principle. Namely, differences in the observed frequency of alternative community structures might have

\footnotetext{
Glossary

Adaptive selection: a selection process (such as neo-Darwinian natural selection) that can cause adaptation to a local environment, because fitness differences result from an interaction between phenotypes and that shared local environment.

Environment (of a system): all local factors external to the system, biotic and/or abiotic (depending upon the system definition).

Feasibility: the existence of an equilibrium point or attractor such that all species in the system can coexist.

Intrinsic system properties: the attributes and qualities of the system as a whole, such as patterns in and magnitudes of interactions and the densities of participating species.

Nonadaptive selection: any selective process that does not act to adapt the members of the population on which it acts to a specific local environment. System: the particular unit of interest, typically involving multispecies interactions but dependent upon the ecological scale of the study. At macroecological scales, we typically refer to systems as multispecies interaction networks (e.g., food or plant-pollinator webs).

Systemic selection: nonadaptive selection on systems based on their intrinsic system properties.
} 
To illustrate the idea of nonadaptive selection, consider an intraguild predation community depicted in Figure I with four slightly different interaction strength configurations. For simplicity, we assume the interactions match the simple Lotka-Volterra model assumed in [3] (e.g., no intraspecific interference competition in the consumers, etc.). Thicker arrows indicate stronger interactions as defined, for example, by the amount of energy flowing from a prey population to each predator. We could expect to obtain each of these configurations in several ways. For example, interaction strengths may change as species identities change through speciation, or the combination of extinction and invasion. Or, they could change from adaptive coevolution among the species, due to variation over space, time, or within populations, or to behavioral changes in individual foraging decisions. However, while many such processes could lead to any of the four configurations, feasibility constraints dictate that we are nonetheless likely to observe one of these four configurations more often in nature than the other three $[4,75]$.
That is, when the intraguild predator and prey are equally efficient competitors for the shared resource (Figure IA), we expect the intraguild prey to be driven towards exclusion because the intraguild predator has the advantage of consuming its competition. Likewise, the intraguild prey will be driven towards exclusion even more strongly when the intraguild predator is the superior competitor (Figure IB), or when the effects of the intraguild predator on the intraguild prey is stronger (Figure IC). Although differences in mortality rates and the presence of alternative resources can weaken and, to some degree, counteract these feasibility requirements, it is when the intraguild prey is the superior competitor (Figure ID) that we are most likely to observe all species coexisting.

Therefore, we can say that communities A-C in Figure I are systemically selected against. Furthermore, because such selection is a result of the properties of the community (i.e., the configuration of interaction strengths) and not due to its particular environment, this selection is nonadaptive (Box 2).
(A)

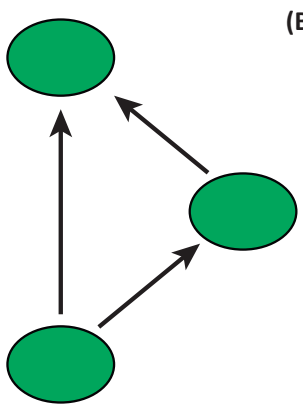

(B)

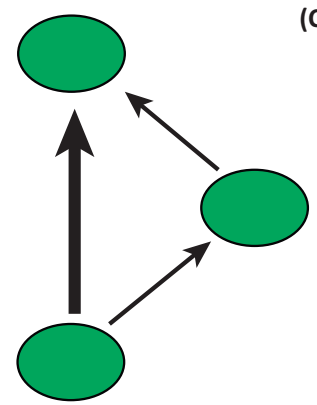

(C)

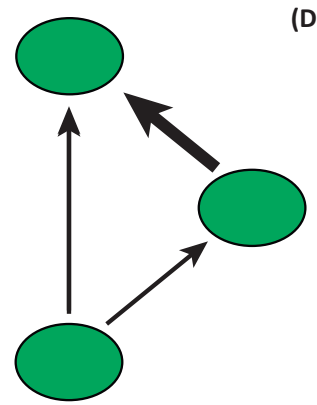

(D)

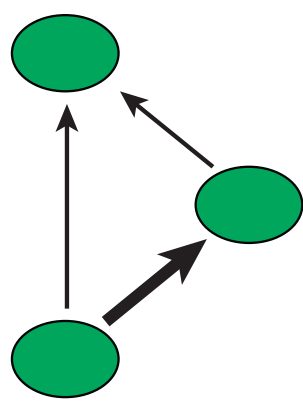

$\overline{T R E N D S}$ in Ecology \& Evolution

Figure I. Four hypothetical intraguild predation communities, at the top is the intraguild predator, the middle is the intraguild prey, and the bottom is the shared resource. Arrows point in the direction of energy flow and their width represents interaction strength.

little to do with the particular characteristics of the local environments in which such systems exist. Instead, intrinsic system properties determining feasibility and stability underlie the relative success or persistence of different configurations, and they can do so more or less independently of local environmental variation. We expect that such nonadaptive systemic selection can produce many of those recurrent ecological patterns that have been observed in nature over large scales of space and time.

Systemic selection can be a powerful yet simple explanation of many ecological patterns, including ecological allometries, species interaction strengths, food-web metrics, and macroevolutionary patterns (e.g., $[5,6]$ ). This intellectual protocol for understanding ecological patterns has long been implicit in community ecology $[7,8]$, but recent advances in theory suggest that it is time to make this principle more explicit and formal. Here, we start with an overview of systemic selection, in the hopes of generating a 'search image' for this type of process in ecology. Then, we dive deeper into (mostly) community ecology to explore the role of systemic selection on stability in connecting local scales to macroscales.

\section{Systemic selection is nonadaptive}

Biologists are used to thinking of 'selection' as a process that explains the adaptation of individuals to local conditions, through a selection regimen based on the interactions of phenotypes with a shared environment. However, macroscale biological systems, such as communities, can also change by the preferential loss of internal configurations that are relatively unstable. This elimination of the unstable is a type of long-term selective process, but one where the selection regimen, instead of being based on an interaction of the biological system or its components with local conditions, derives primarily from fundamental properties of the system itself; the process does not 'see' the local conditions and neither is it activated by environmental change (Box 2).

A theory that is based on such a systemic selection process can be thought of as 'nonadaptive', in the sense that the selection process it describes does not adapt the system to local conditions, even though (if working alone) it changes the system in a given direction. We use 'adaptation' as is commonly understood, illustrated by Vermeij's definition (p. 364 in [9]): ' . . as a heritable attribute of an entity that confers advantages in survival and reproduction of that entity in a given environment.' Vermeij goes on to say that these 'advantages' are 'context dependent'. A trait that is an adaptation in one environment might not be in a different one. By contrast, what we refer to as systemic selection involves 'advantages' that are context independent.

Of course, the properties of these systems likely change in significant ways by ordinary adaptive selection and/or 
Scientists use the term 'adapt' and its derivatives in various ways. For our purposes, the process of 'adaptation' can be most usefully considered as 'adaptation to a specific locally shared environment'. This is the most common usage in evolutionary biology. In emphasizing that, for some systems, there are systemic selective processes that impose the same selective forces regardless of variation across local environments, we highlight the fact that these processes are selective, but for reasons that do not involve adaptation to local conditions.

This perspective contrasts with a more conventional approach toward the evolution of macroscale biological systems, accomplished by expanding hierarchically the theory of adaptation by natural selection by identifying a hierarchy of units of selection [76]. 'Individual' and 'population' are replaced with 'population' and 'community' (or 'community' and 'population-of-communities'), respectively, and analogous properties of these entities that correspond to key elements of organismic natural selection (e.g., reproduction, heritability, fitness, adaptation, or environment) are identified. It has long been recognized that such a theory of the adaptation of higher-level entities to their environments is logically possible, although not without significant issues [76-79] (J. Khai Tran, PhD thesis, Stony Brook University, 2011).

In this view, feasibility and stability serve as an important component of community fitness and are considered to depend on the environment. For example, the environment can influence the strength and type of interactions between species. Moreover, overall community fitness also relies on 'reproduction' in terms of a community colonizing new areas or influencing the composition of other

coevolution among their members, and by processes such as migration and drift, tending to generate diversity within and among systems (e.g., communities). However, because systemic selection operates on intrinsic system properties that do not vary with the environment, it can, if not significantly overwritten by other processes, have a major role in explaining the evolution and stable properties of macroscale biological systems. Such nonadaptive processes tend to promote similarity rather than diversity among ecological systems. A book-length treatment that explores nonadaptive selection more generally is currently in preparation by Damuth and Ginzburg.

\section{Constraints on attack rates and handling times}

Nonadaptive systemic selection can act on whole communities and ecosystems, but we begin by describing how it can also act on the pairwise interactions of species. Each species in an interacting pair brings with it attributes that may affect the feasibility and stability of its interaction. Species pairs with attributes that lead to extinction because they generate highly unstable dynamics are not likely to be observed, compared with species pairs whose attributes permit stable dynamics.

The well-studied Rosenzweig and MacArthur model [10] provides a conceptual foundation for understanding how consumer traits, such as attack rates and handling times, influence oscillatory dynamics in a broad array of predator-prey models [11]. In this model, oscillations increase because the handling time induces positive feedback in the per capita growth rate of the resource species, which enables prey to increase in abundance; the ensuing increase in the predator population leads to prey overexploitation and a decline in the abundance of the prey followed by the predators [12]. Oscillatory dynamics are more likely to occur when prey are weakly self-limited (e.g., high communities through propagule pressure. Although the process is more nuanced, in oversimplified terms, adaptive selection at the community level eliminates community types that on average do not persist long enough to produce a like community (i.e., absolute fitness less than 1). This is likely the fate of infeasible and highly unstable configurations, barring a reproductive rate high enough to compensate. Furthermore, community selection can favor one stable community type over another according to their relative fitnesses. Similarity among existing communities is explained as the consequence of convergence (limited ways to solve a problem), with the evolutionary process being higher level adaptive selection.

Despite its logical appeal, a consensus has not emerged about how to put in practice such a theory so that it both avoids conceptual difficulties and provides rewarding insights into community evolution. From the perspective of nonadaptive selection, a stable community is not necessarily a community that is 'better adapted' to its specific environment. Exploring nonadaptive selection does not require resolving issues about defining local adaptation at the community level. A useful analogy from the physical sciences illustrates this point. Protons, the nuclei of hydrogen atoms, have never been observed to decay while the most stable isotope of the radioactive element francium is unstable (short half-life). Hydrogen is the most abundant element in the universe, whereas francium is a laboratory curiosity. Yet, we would be unwilling to say that hydrogen is better adapted to the universe. Eventually, a fruitful adaptive theory of community evolution, as a counterpart to the nonadaptive processes we discuss here, might be possible.

carrying capacity) and predators have high attack rates and long handling times [11].

Oscillations in population abundances predispose species, and the interaction modules in which they occur, to stochastic extinctions during phases of low abundance. The disproportionate extinction of interactions exhibiting large amplitude oscillations imposes selection at the population level. The expectation that species with oscillatory dynamics should therefore be uncommon in nature [13] is borne out by analyses of time-series data, which show only approximately $30 \%$ of species with evidence of consumer-driven cycles [14]. However, such cycles are particularly common in tightly coupled interactions between specialist predators and their prey [15].

Given that systemic selection tends to cull predatorprey interactions in which oscillations lead to periods of low abundances, the attack rates and handling times of predators should be inversely related; that is, high attack rates and short handling times (greater overexploitation), low attack rates and long handling times (low exploitation potential), or low attack rates and short handling times (short time delay), which dampen oscillations.

Johnson and Amarasekare [12] establish the veracity of this prediction through analysis of the attack rates and handling times of 57 functional response experiments as well as the functional responses and self-limitation strength of two specialist parasitoids and their herbivorous insect host (Figure 1A) [16]. Likewise, estimating the attack rates and handling times of two generalist intertidal whelk predators feeding on 7-33 different prey species [4], Novak (PhD thesis, University of Chicago, 2008) showed that 181 pairwise interactions measured across six sites exhibited combinations of attack rates and handling times consistent with the predicted inverse relation (Figure 1B).

Thus, empirical evidence supports the theoretical prediction that the stability of consumer-resource interactions 


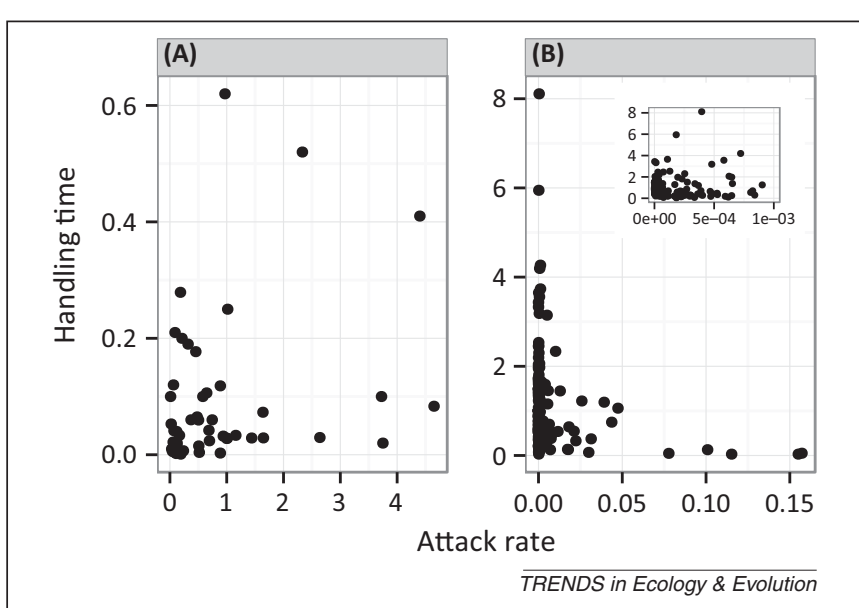

Figure 1. The empirical relation between handling time and attack rates. (A) Published attack rates $\left(\right.$ day $^{-1}$ ind $\left.^{-1}\right)$ and handling times $\left(\right.$ day $\left.^{-1}\right)$ for 57 species; see [12] for details. (B) Empirical relation between the species-specific handling times (days) and per capita attack rates (prey eaten pred ${ }^{-1}$ prey $^{-1} \mathrm{~m}^{-2}$ day $^{-1}$ ) of two predatory whelk species feeding on their prey $(n=181$; M. Novak, PhD thesis, University of Chicago, 2008). Inset shows the relation at low attack rates.

constrains attack rate and handling time combinations to be either low and/or inversely related. An important task for future work will be to examine the simultaneous operation of systemic selection due to instability, and ongoing natural selection within each of the coevolved species that influence attack rates and handling times [4].

Similar results have been found based on models of entire food webs. Nonadaptive systemic selection, through stability and feasibility constraints, can restrict observed attack rates in a community to values where new consumers invade but do not overexploit their resources. The possibility of such selection among otherwise unconstrained attack rates during food-web composition turnover was first demonstrated in a model by Rossberg et al. [17].

Community turnover is essential to this mechanism, because this enables species with a lower attack rate compared with current residents to invade, at least when residents and invaders sufficiently differ from each other to not strongly compete through shared resources and consumers. This could result from the immigration of individuals from a large species pool. Variants with lower attack rates can have higher chances of establishing in a food web and persisting compared with those with higher attack rates, even though their invasion fitness [18] is lower, if they avoid overexploiting and extinguishing their main resources.

A detailed analysis of this phenomenon must account for the distribution of trophic interaction strengths among consumer-resource pairs, and trophic niche widths [19]. Thus, this analysis identifies an 'optimal' degree of dietary diversity for consumers, where the risk of resource overexploitation is minimized, matching well the dietary diversity found for marine fish $[19,20]$. Another line of evidence for systemic selection of attack rates is that aquatic size spectra (the distribution of community biomass over individuals of different sizes [21]) should theoretically have the observed power-law forms only if mean attack rates are constrained to appropriate values, at least to an order of magnitude [22].
Thus, systemic selection at the community level, over long timescales, can lead to predictable changes of attack rates and niche widths, because these traits have similar ecological implications across communities of different composition. Traits with dynamical implications that depend more sensitively on community composition, such as some specialized foraging or defense strategies [23], might be less likely to exhibit predictable patterns across space or time.

\section{Community modules}

The structure of ecological networks strongly differs from random, particularly at the level of small-scale structures (network modules or motifs), considered to be the 'building blocks' of networks. Network patterns can be altered by changing the number of species through invasion, speciation, and extinction, or by changing the way species interact (e.g., by changing trophic relations or interaction strengths).

In food webs, some modules are greatly over-represented (e.g., the tritrophic chain) [24-26]. The preponderance of certain motifs could reflect their dynamical properties $[25,27,28]$, and simulations indeed show that motif profiles have strong influence on network dynamics. If different food web configurations vary in stability, systemic selection should cull unstable configurations $[2,7]$. Such systemic selection could operate through two intertwined processes: network assembly and dynamical pruning $[29,30]$.

The degree to which a community is stable can be determined through simulation [28], given assumptions about the equations governing dynamics (e.g., Box 3). The network motifs that are observed in food webs are also the structures with the greatest probability of being stable (Figure 2) [25]. Aside from motif patterns, food webs exhibit a pattern of short food chains that can be explained by stability constraints [8,31]. Food webs built with longer chains are less stable than those with shorter chains [32]. These results match what we expect from systemic selection against instability.

Nonadaptive systemic selection can act on networks via dynamical pruning, favoring configurations that are more likely to lead to system persistence [31]. Similar nonrandom motif profiles could also arise by network assembly, the effects of which have been found in both mutualistic and trophic networks [33-37]. Only by developing strong models of network assembly can one separate the effect of dynamical pruning from that of network growth and development. Assembly might not only involve systemic selection, but also include other processes (e.g., priority effects or constraints on colonization).

\section{Limits of feasible coexistence}

When modeling large numbers of interacting species with arbitrary, yet biologically plausible, combinations of traits and interactions, it is difficult to achieve coexistence of all species. During simulations, at least some species will inevitably decline to zero abundance. Often this is because there is no equilibrium where all species can coexist (infeasibility) rather than any existing equilibrium point being unstable. 


\section{Box 3. Modeling species interactions}

Usually, interactions within food webs are modeled with generalized Lotka-Volterra equations. However, although these equations can be acceptable in some conditions, they do not apply universally. The Lotka-Volterra functional response model assumes random and homogeneous mixing of prey and predator individuals, and that predators are rare enough to avoid interference. Empirical evidence and theoretical considerations suggest that it is necessary to use different models, particularly with incorporation of predator interference. Extra care must be taken when using non-Lotka-Volterra interactions in food-web models to ensure that the generalized mathematical expression remains logically consistent [80]

An alternative to Lotka-Volterra functional response models, the donor-control model, is a simple case of ratio dependence that has often been observed. This type of interaction might result not only from nonadaptive selection, but also from complex mechanistic models that incorporate conventional adaptive selection [81]. We can generalize the donor-control model to a multispecies case, where several predator and/or prey species interact. For two prey species having a common predator, one prey species can lead to indirect exclusion of the other species (apparent competition). In the case of two predators alone competing for a single prey species, one species is always excluded by the other, and this is true often even in the presence of a top predator. That is, a keystone effect is never observed and the configuration collapses to a three-level food chain. Given donor control, simple food chains will persist or not because of basal production, not because of top-down effects. Intraguild predation can, in some instances, help maintain more complex structures.

Feasibility constraints can be powerful modes of systemic selection. Models have shown that the number of species that can coexist in equilibrium communities (with no direct competition within or between species $[38,39]$ ) cannot be greater than the number of resources mediating indirect exploitative competition [40,41]. When there are more species than resources, some will invariably outcompete the others, resulting in a final community with, at most, one species per resource.

Only recently has it become clear [19] that these constraints can become tighter when the competing species

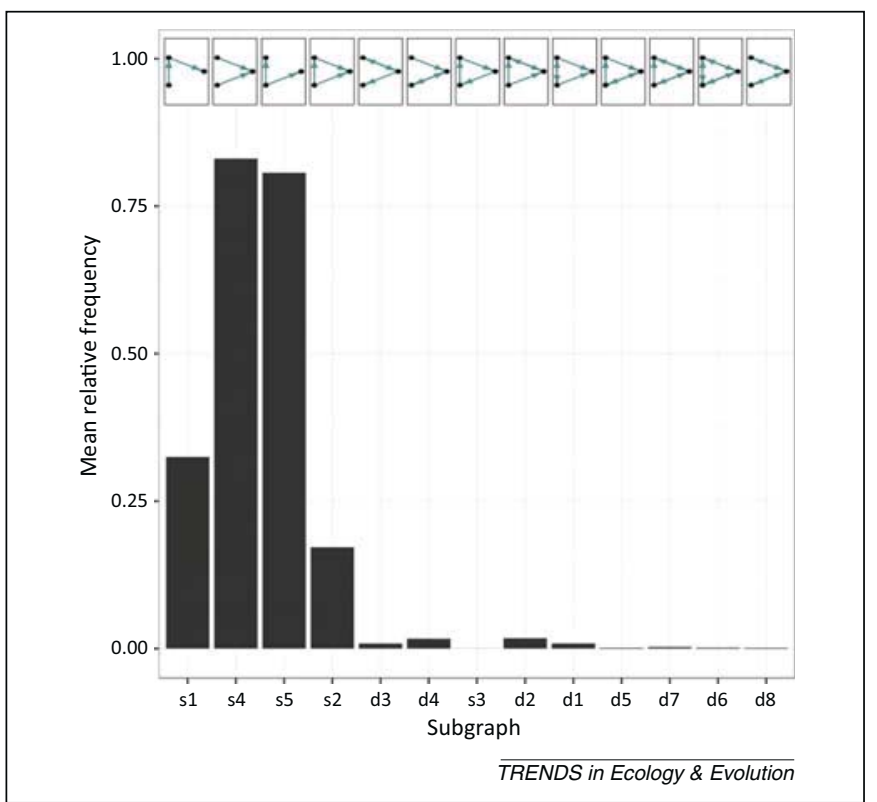

Figure 2. The mean relative frequency of the 13 three-node subgraphs (pictured at the top of the plot) in 50 empirical food webs ordered by their stability. For more details on methods and data, see [25]. target resources selectively, so that most species have just a few, but strong, competitors. In two-level food webs where a community of consumer species feeds on, and competes for, members of a community of resource species, and similarly for host-parasite communities, it has been shown that feasible coexistence of the consumer species becomes unlikely if their number exceeds half the number of resource species [19]. Beyond this point, small changes in model parameters lead to large changes in abundances. While coexistence of more consumers might then still be possible, it would require a degree of fine-tuning of parameters that is unlikely.

When several trophic levels are stacked upon each other, the feasibility constraint tightens even further, and only approximately one-third of the number of species at one trophic level are likely to coexist in the next higher trophic level. The potential explanatory power of this mode of systemic selection matches the consistent observations of a 1:2 species-richness ratio in host-parasite communities $[19,42,43]$ and 1:3 richness ratios at adjacent trophic levels in multitrophic food webs [19,44-46] (Figure 3).

Although other potential mechanisms producing correlations of richness across trophic levels have been proposed $[44,45,47]$, none to date predict specific values for the richness ratio. For size-structured food webs where the adult body sizes of consumers and resources are related by a characteristic predator-prey mass ratio (PPMR), systemic selection arising from feasibility constraints implies a decline of species richness with body mass as a power law with exponent $-\log (3) / \log (\mathrm{PPMR}) \approx-0.2$, consistent with numerical and empirical observations [48-51].

Systemic selection at the community level and beyond Systemic selection at the scale of entire communities should result in patterns in ecological systems that are more feasible and stable than expected at random. One way to characterize a community is the species by species community matrix, whose off-diagonal elements quantify interspecific interactions, specifically how fast the population of one species changes as a result of small deviations of another species from its equilibrium abundance. The

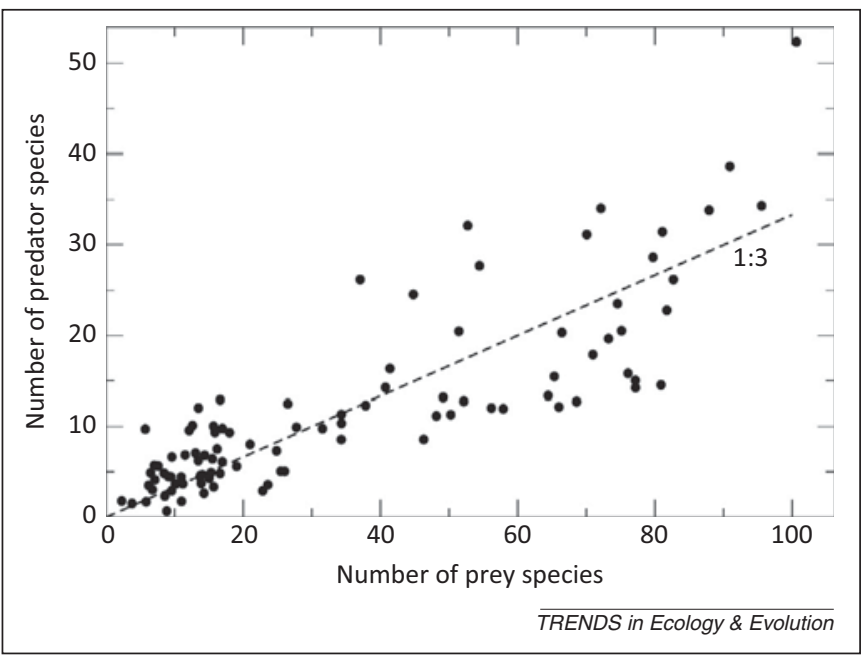

Figure 3. Predator-prey richness ratios in freshwater invertebrate communities after [44], compared with the predicted ratio of 1:3 (broken line). 
diagonal entries describe the strengths of intraspecific density dependencies.

Even without knowing the magnitude of these interactions, one can still address the question of which kinds of structure have a selective advantage (i.e., are more stable), by comparing structures of interactions formalized as signed directed graphs. Such a graph describes the community matrix in a magnitude-independent way; that is, only by the pattern of pluses, minuses, and zeros within the matrix. Patterns of interactions that are sign stable (as magnitude-independent community matrices) are robust, in that environmental changes affecting strengths (but not signs) of interactions cannot flip a system from stability to instability. Thus, mathematical results on criteria for sign stability (which are known, [52-55]) provide qualitative predictions about patterns in community structure that should result.

Sign-stable configurations in nature are rare: simple trophic chains (without omnivory) or a few chains linked by amensalistic or commensalistic links with no cycles. Horizontally structured communities (i.e., those without trophic interactions) cannot be sign stable, but can be stable depending on the strength of interactions. The utility of a particular concept of stability of a system depends on the level of detail in the system description. When a food chain is used to represent a series of stable, horizontally structured aggregates of species, then the chain will be sign stable if and only if omnivory is absent. In a sense, one should expect a sign-stable community of this kind to be selected because it will remain feasible despite variation in parameter values.

However, omnivorous links are frequently observed in trophic networks [56-58]. Therefore, one must look more closely to identify in community matrices a signature of nonadaptive systemic selection through stability constraints. Given two networks with omnivorous links that are both stable (i.e., will return to equilibrium following a small perturbation) the matrix flower (Box 4) can be used to determine which is more likely to be eliminated because of instability.

We can also try to determine how large the feasibility domain (parameter space compatible with coexistence) is and how this domain is modulated by network architecture and interaction strengths. In a competition system, for instance, we can show that the lower the level of interspecific competition, relative to intraspecific, the larger the domain of feasibility [59]. For mutualistic interactions, with a given number of species, links, and distribution of interaction strengths, feasibility domains increase with network nestedness [60]. A community pattern with a larger tolerance to perturbations in biotic and abiotic factors is, by definition, more structurally stable and, under the hypothesis of systemic selection, should be more frequently observed. As predicted, nestedness is commonly found in plant-frugivore and plant-pollinator networks (both mutualistic) [61].

The idea that something analogous to natural selection acts on ecosystems has been around for almost as long as

\section{Box 4. The matrix flower}

In addition to the famous concept of Lyapunov stability with its wellknown matrix criterion, there are several other, less well-known stability concepts defined in matrix terms: D- and aD-stability, signstability, Volterra dissipativeness, and others. These stability concepts deal with stability despite various kinds of uncertainty or perturbation. However, few such concepts have clear characterizations via the matrix structure and/or elements, and some just have definitions, with only necessary or sufficient conditions for their verification known. Logical inclusion relations (Venn diagrams) among the special stability subsets in a general space of real $n \times n$ matrices help verify particular properties of a given matrix. Adding a few more conventions to a standard Venn diagram turns it into a 3D matrix flower [82], whose 'petals' represent the subsets of matrices with particular stability properties (Figure I).

Two, relatively small, petals of sign-stable matrices are located in the very 'core' of the flower, while the other stability petals signify the lower levels in nonadaptive selection.

We should keep in mind that having the most stable pattern in interspecific interactions is only one side of the problem. The other side is the feasibility of the community, that is, the existence of an equilibrium point where all species have strictly positive abundances. Illustrating that feasibility is as crucial as the stability constraint, consider the simple example given by a set of species competing for resources. Moreover, assume that the interspecific interaction matrix has been derived from species overlap in a niche space [83]. In this case, the matrix belongs automatically to the petal of being Volterra dissipative. This implies that the community is automatically globally stable as long as there exists a feasible equilibrium point. Therefore, the only remaining question is whether the system will be feasible. We can show that there exists a specific domain in the space of carrying capacities (or intrinsic growth rates), in which the community is feasible $[55,84]$. An open question is how these ideas translate into systems where coexistence arises because of nonlinear feedbacks (e.g., Armstrong-McGehee effects [85,86]).

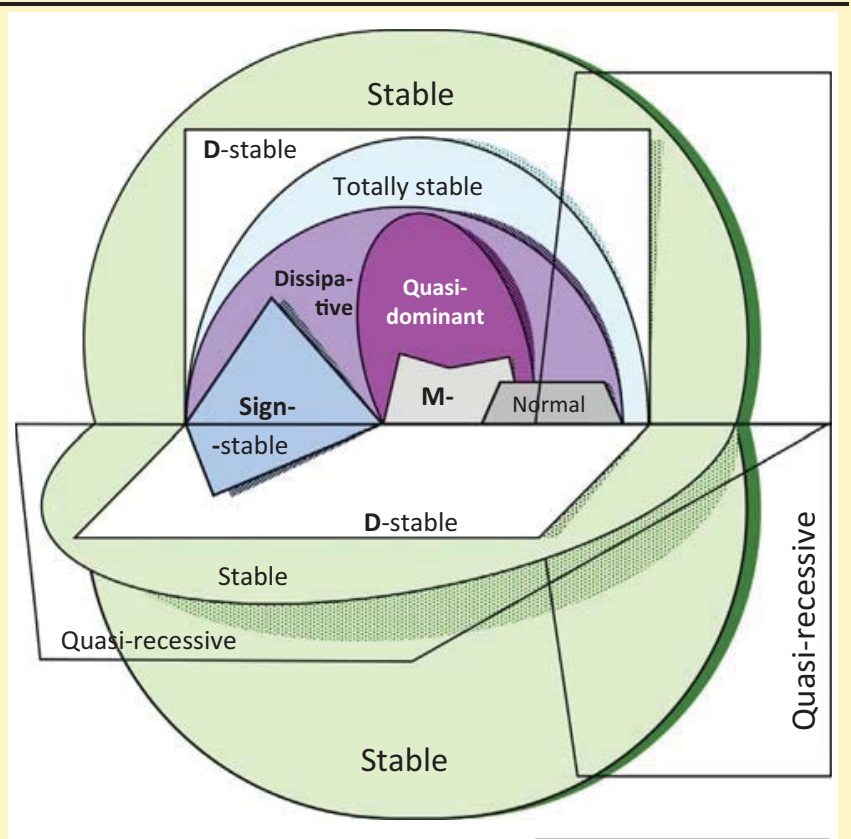

TRENDS in Ecology \& Evolution

Figure I. Logical relations among different types of matrix stability. Adapted from [82]. 
the idea that natural selection acts on species. For example, Lotka [62] claimed that natural selection maximizes the total energy flux through an ecosystem. Lotka's argument was based on Boltzmann's [63] statement that entropy, and not raw materials, is the limiting factor for all organisms. This intuition provided the foundation for a distinctive school of ecosystem ecology (e.g., [64,65]), whose ideas are not currently widely accepted (although this does not imply that they are wrong).

Taking a different approach, Tansley [66] argued that 'a kind of natural selection' will tend to favor more stable ecosystems. This view has remained widespread in ecological theory. However, empirically evaluating the degree to which a particular selective mechanism might have shaped ecosystem properties requires knowledge of transient states that have been selected against. Our sample of observed ecosystems is unfortunately 'length biased': because most observations come from single time points, ecosystems that are more persistent are likely to be systematically over-represented, relative to the true distribution of historical persistence times. If ecosystem structure is shaped over time by stability constraints, we should expect unbiased samples (taken through time at fixed points of space) to differ systematically from extant ecosystems, which would represent the stable end products of historical processes.

There have occasionally been claims that natural selection can operate between adjacent 'meta-ecosystems', such that the most stable units leave the most descendants (e.g., [67]). However, it seems likely that such mechanisms will only work if ecosystems replicate themselves in their entirety by occupying new units of space. Nonadaptive selection does not require an analog of reproduction at higher levels of organization than the individual, but does depend upon persistence of definable configurations of species across time.

\section{Social systems}

Here, we have mainly focused on community ecology. Yet, processes of nonadaptive systemic selection might also be relevant in explaining many other biological patterns. Consider, for instance, structure within populations, in particular, the formation of animal social systems. In small groups in many species (from wasps to human children), dominance hierarchies often reveal linear structures. In a linear hierarchy, one animal dominates all others, being aggressive toward them without receiving aggression in turn. A second dominates all but the top, and so on. All possible subgroups of three individuals (triads) in a group must have transitive configurations of attack (A attacks B, B attacks C, and A attacks C) for a hierarchy to be linear. If a hierarchy is not linear, then at least one triad has intransitive attack configurations (A attacks B, B attacks C, and C attacks A).

Traditionally, researchers have explained this pattern of organization using individual-based, adaptive models. They have proposed that differences in individual attributes (e.g., size, weaponry, or aggressiveness) determine ranks in the hierarchy $[68,69]$. Or, they have suggested that feedback from winning or losing contests during group formation determines individual ranks [70,71]: an individual winning a contest increases its probability of winning another contest with a new individual, and an individual losing a contest increases its probability of losing. However, both experimental and analytical work [72,73] demonstrate that these simple biological mechanisms of differences in attributes, or winner and loser effects, are unable account for the observed structure of linear hierarchies. Instead, they are better explained by the differential stability rates of the building blocks of the structures.

In analyses of dynamical, peck-by-peck records of chickens forming hierarchies, Chase and Lindquist [74] discovered that intransitive configurations of attacks were unstable, usually lasting only for brief periods of time before changing to transitive ones. By contrast, transitive configurations lasted over 20 times as long as intransitive ones on average. They hypothesized that linear hierarchies are common across many species because, as in chickens, when intransitive configurations of attacks appear, they convert quickly to transitive ones, ensuring that hierarchy structures are almost always linear. This rule (transitive configurations are more stable than intransitive ones), plus the rule that all individuals interact, suffices to generate hierarchies that are almost always linear.

\section{Concluding remarks}

A major focus of evolutionary biology is upon processes that create and maintain diversity: how organisms become adapted to their various local environments (including other organisms). However, especially with regard to multispecies interaction systems, it is also important to understand the processes that result in similarity among systems. Here, we have argued that, because of system dynamics, the deck is stacked in favor of some (stable and feasible) configurations and against others, so that what one observes is a nonrandom distribution of system architectures, based on intrinsic properties shared across systems. There is a resurgence of interest in stability and selective processes in shaping ecological communities and their component structures (food webs, food chains, etc.). New theoretical approaches and computational resources reveal the potential significance of 'nonadaptive' systemic selection processes and highlight key attributes promoting stability. At the same time, stability and feasibility are themselves complex concepts that have not been fully explored. Identification of stability criteria for communities and community modules might depend significantly on how researchers model smaller-scale consumer-resource interactions. Nevertheless, it is likely that systemic selection processes (processes of pruning away unstable configurations) will increasingly be seen to underlie many recurrent structural features of biological systems, within which the more familiar processes of local adaptation operate.

\section{Acknowledgments}

This work is the outcome of a workshop gathering all of the authors at the Centre Interfacultaire Bernoulli of the Ecole Polytechnique Fédérale de Lausanne in Switzerland (EPFL), with funding of the Swiss National Science Foundation (http://cib.epfl.ch/prog.php?id=\%271000000027\%27).

\section{References}

1 Schoener, T.W. (2011) The newest synthesis: understanding the interplay of evolutionary and ecological dynamics. Science 331, 426-429 
2 Kondoh, M. (2008) Building trophic modules into a persistent food web. Proc. Natl. Acad. Sci. U.S.A. 105, 16631-16635

3 Holt, R.D. and Polis, G.A. (1997) A theoretical framework for intraguild predation. Am. Nat. 149, 745-764

4 Novak, M. (2013) Trophic omnivory across a productivity gradient: intraguild predation theory and the structure and strength of species interactions. Proc. Biol. Sci. 280, 20131415

5 Damuth, J. (2007) A macroevolutionary explanation for energy equivalence in the scaling of body size and population density. Am. Nat. 169, 621-631

6 Ginzburg, L.R. et al. (2010) The May threshold and life-history allometry. Biol. Lett. 6, 850-853

7 Pimm, S.L. (1982) Food Webs, University of Chicago Press

8 Pimm, S.L. and Lawton, J.H. (1977) Number of trophic levels in ecological communities. Nature 268, 329-331

9 Vermeij, G.J. (1996) Adaptations of clades: resistance and response. In Adaptation (Rose, M. and Lauder, G., eds), pp. 363-380, Academic Press

10 Rosenzweig, M.L. and MacArthur, R.H. (1963) Graphical representation and stability conditions of predator-prey interactions. Am. Nat. 97, 209-223

11 Rosenzweig, M.L. (1971) Paradox of enrichment: destabilization of exploitation ecosystems in ecological time. Science 171, 385-387

12 Johnson, C.A. and Amarasekare, P. (2015) A metric for quantifying the oscillatory tendency of consumer-resource interactions. Am. Nat. 185 , 87-99

13 Gilpin, M.E. (1975) Group Selection in Predator-Prey Communities, Princeton University Press

14 Kendall, B.E. et al. (1998) The macroecology of population dynamics: taxonomic and biogeographic patterns in population cycles. Ecol. Lett. $1,160-164$

15 Murdoch, W.W. et al. (2002) Single-species models for many-species food webs. Nature 417, 541-543

16 Kidd, D. and Amarasekare, P. (2012) The role of transient dynamics in biological pest control: insights from a host-parasitoid community. J. Anim. Ecol. 81, 47-57

17 Rossberg, A.G. et al. (2008) The top-down mechanism for body-massabundance scaling. Ecology 89, 567-580

18 Metz, J. (2008) Fitness. In Encyclopedia of Ecology (Vol. 2) (Jørgensen, S.E. and Fath, B.D.,eds) pp. 1599-1612, Elsevier

19 Rossberg, A.G. (2013) Food Webs and Biodiversity: Foundations, Models, Data, Wiley

20 Rossberg, A.G. et al. (2011) Universal power-law diet partitioning by marine fish and squid with surprising stability-diversity implications. Proc. R. Soc. B 278, 1617-1625

21 Sheldon, R.W. and Parsons, T.R. (1967) A continuous size spectrum for particulate matter in the sea. J. Fish. Res. Board Canada 24, 909-915

22 Rossberg, A. (2012) A complete analytic theory for structure and dynamics of populations and communities spanning wide ranges in body size. Adv. Ecol. Res. 46, 429-522

23 Nagelkerke, L.A.J. and Rossberg, A.G. (2014) Trophic niche-space imaging, using resource and consumer traits. Theor. Ecol. 7, 423-434

24 Stouffer, D.B. et al. (2007) Evidence for the existence of a robust pattern of prey selection in food webs. Proc. R. Soc. B 274, 1931-1940

25 Borrelli, J.J. (2015) Selection against instability: stable subgraphs are most frequent in empirical food webs. Oikos Published online March 6 , 2015. http://dx.doi.org/10.1111/oik.02176

26 Bascompte, J. and Melián, C.J. (2005) Simple trophic modules for complex food webs. Ecology 86, 2868-2873

27 Prill, R.J.et al. (2005) Dynamic properties of network motifs contribute to biological network organization. PLoS Biol. 3, 1881-1892

28 Allesina, S. and Pascual, M. (2008) Network structure, predator-prey modules, and stability in large food webs. Theor. Ecol. 1, 55-64

29 Solé, R.V. and Valverde, S. (2006) Are network motifs the spandrels of cellular complexity? Trends Ecol. Evol. 21, 419-422

30 Camacho, J. et al. (2007) Quantitative analysis of the local structure of food webs. J. Theor. Biol. 246, 260-268

31 Ulanowicz, R.E. et al. (2013) Limits on ecosystem trophic complexity: insights from ecological network analysis. Ecol. Lett. 17, 127-136

32 Borrelli, J.J. and Ginzburg, L.R. (2014) Why there are so few trophic levels: selection against instability explains the pattern. Food Webs 1, 10-17
33 Eklöf, A. et al. (2012) Relevance of evolutionary history for food web structure. Proc. R. Soc. B 279, 1588-1596

34 Stouffer, D.B. et al. (2012) Evolutionary conservation of species' roles in food webs. Science 335, 1489-1492

35 Rezende, E.L. et al. (2007) Non-random coextinctions in phylogenetically structured mutualistic networks. Nature 448, 925-928

36 Cattin, M. et al. (2004) Phylogenetic constraints and adaptation explain food-web structure. Nature 427, 835-839

37 Rossberg, A.G. et al. (2006) Food webs: experts consuming families of experts. J. Theor. Biol. 241, 552-563

38 Mcpeek, M.A. (2012) Intraspecific density dependence and a guild of consumers coexisting on one resource. Ecology 93, 2728-2735

39 McPeek, M.A. (2014) Keystone and intraguild predation, intraspecific density dependence, and a guild of coexisting consumers. Am. Nat. 183, E1-E16

40 MacArthur, R. and Levins, R. (1964) Competition, habitat selection, and character displacement in a patchy environment. Proc. Natl. Acad. Sci. U.S.A. 51, 1207-1210

41 Rescigno, A. and Richardson, I.W. (1965) On the competitive exclusion principle. Bull. Math. Biophys. 27, 85-89

42 Santos de Araújo, W. (2011) Can host plant richness be used as a surrogate for galling insect diversity. Trop. Conserv. Sci. 4, 420-427

43 Wright, M.G. and Samways, M.J. (1998) Insect species richness tracking plant species richness in a diverse flora: gall-insects in the Cape Floristic Region, South Africa. Oecologia 115, 427-433

44 Jeffries, M.J. and Lawton, J.H. (1985) Predator-prey ratios in communities of freshwater invertebrates: the role of enemy free space. Freshw. Biol. 15, 105-112

45 Warren, P.H. and Gaston, K.J. (1992) Predator-prey ratios: a special case of a general pattern? Philos. Trans. R. Soc. B 338, 113-130

46 Jennings, S. et al. (2002) Use of size-based production and stable isotope analyses to predict trophic transfer efficiencies and predatorprey body mass ratios in food webs. Mar. Ecol. Prog. Ser. 240, 11-20

47 Spencer, M. (2000) Are predators rare? Oikos 89, 115-122

48 Jonsson, T. et al. (2005) Food webs, body size, and species abundance in ecological community description. Adv. Ecol. Res. 36, 1-84

49 ICES (2013) Report of the Working G-roup on the Ecosystem Effects of Fishing Activities (WGECO), ICES

50 ICES (2014) Report of the Working G-roup on the Ecosystem Effects of Fishing Activities (WGECO), ICES

51 Reuman, D.C. et al. (2014) The marine diversity spectrum. J. Anim. Ecol. 83, 963-979

52 May, R.M. (1973) Qualitative stability in model ecosystems. Ecology $54,638-641$

53 Jeffries, C. (1974) Qualitative stability and digraphs in model ecosystems. Ecology 55, 1415-1419

54 Jeffries, C. et al. (1987) Qualitative stability of linear systems. Linear Algebra Appl. 87, 1-48

55 Logofet, D. (1993) Matrices and Graphs, CRC Press

56 Thompson, R.M. et al. (2007) Trophic levels and trophic tangles: the prevalence of omnivory in real food webs. Ecology 88, 612-617

57 Williams, R.J. and Martinez, N.D. (2004) Limits to trophic levels and omnivory in complex food webs: theory and data. Am. Nat. 163, 458468

58 Arim, M. and Marquet, P.A. (2004) Intraguild predation: a widespread interaction related to species biology. Ecol. Lett. 7, 557-564

59 Saavedra, S. et al. (2014) How structurally stable are global socioeconomic systems? J. R. Soc. Interface 11, 20140693

60 Rohr, R.P. et al. (2014) On the structural stability of mutualistic systems. Science 345, 1253497

61 Bascompte, J. et al. (2003) The nested assembly of plant-animal mutualistic networks. Proc. Natl. Acad. Sci. U.S.A. 100, 9383-9387

62 Lotka, A. (1922) Contribution to the energetics of evolution. Proc. Natl. Acad. Sci. U.S.A. 8, 147-151

63 Boltzmann, L. (1974) Theoretical Physics and Philosophical Problems: Selected Writings, Reidel Publishing Company

64 Odum, H. and Pinkerton, R. (1955) Time's speed regulator: the optimum efficiency for maximum power output in physical and biological systems. Am. Sci. 43, 331-343

65 Ulanowicz, R. (1986) Growth and Development: Ecosystems Phenomenology, iUniverse 
66 Tansley, A.G. (1935) The use and abuse of vegetational concepts and terms. Ecology 16, 284

67 Dunbar, M. (1972) The ecosystem as unit of natural selection. Trans. Connect. Acad. Arts Sci. 44, 111-130

68 Jackson, W.M. and Winnegrad, R.L. (1988) Linearity in dominance hierarchies: a second look at the individual attributes model. Anim. Behav. 36, 1237-1240

69 Holekamp, K.E. and Smale, L. (1993) Ontogeny of dominance in freeliving spotted hyaenas: juvenile rank relations with other immature individuals. Anim. Behav. 46, 451-466

70 Beaugrand, J.P. and Cotnoir, P-A. (1996) The role of individual differences in the formation of triadic dominance orders of male green swordtail fish (Xiphophorus helleri). Behav. Processes 38, 287-296

71 Hsu, Y.Y. and Wolf, L.L. (1999) The winner and loser effect: integrating multiple experiences. Anim. Behav. 57, 903-910

72 Chase, I.D. et al. (2002) Individual differences versus social dynamics in the formation of animal dominance hierarchies. Proc. Natl. Acad. Sci. U.S.A. 99, 5744-5749

73 Lindquist, W.B. and Chase, I.D. (2009) Data-based analysis of winnerloser models of hierarchy formation in animals. Bull. Math. Biol. 71, 556-584

74 Chase, I.D. and Lindquist, W.B. (2009) Dominance hierarchies. In The Oxford Handbook of Analytical Sociology (Hedström, P. and Bearman, P., eds), pp. 566-591, Oxford University Press

75 Kondoh, M. (2003) Foraging adaptation and the relationship between food-web complexity and stability. Science 299, 1388-1391
76 Lewontin, R.C. (1970) The units of selection. Annu. Rev. Ecol. Syst. 1, $1-18$

77 Hull, D.L. (1980) Individuality and selection. Annu. Rev. Ecol. Syst. 11, 311-332

78 Damuth, J. (1985) Selection among 'species': a formulation in terms of natural functional units. Evolution 39, 1132-1146

79 Brandon, R. (1990)Adaptation and Environment, Princeton University Press

80 Arditi, R. and Michalski, J. (1996) Nonlinear food web models and their responses to increased basal productivity. In Food Webs: Integration of Patterns and Dynamics (Polis, G.A. and Winemiller, K.O., eds), pp. 122-133, Chapman \& Hall

81 Arditi, R. and Ginzburg, L.R. (2012) How Species Interact: Altering the Standard View on Trophic Ecology, Oxford University Press

82 Logofet, D.O. (2005) Stronger-than-Lyapunov notions of matrix stability, or how 'flowers' help solve problems in mathematical ecology. Linear Algebra Appl. 398, 75-100

83 MacArthur, R. and Levins, R. (1967) The limiting similarity, convergence, and divergence of coexisting species. Am. Nat. 101, 377-385

84 Svirezhev, Y. and Logofet, D. (1983) Stability of Biological Communities, MIR Publisher

85 Armstrong, R.A. and Mcgehee, R. (1980) Competitive exclusion. Am. Nat. 115, 151-170

86 Abrams, P.A. and Holt, R.D. (2002) The impact of consumer-resource cycles on the coexistence of competing consumers. Theor. Popul. Biol. $62,281-295$ 